\title{
Beneficial Regulation of Elastase Activity and Expression of Tissue Inhibitors of Matrixmetalloproteinases, Fibrillin, Transforming Growth Factor- $\beta$, and Heat Shock Proteins by $P$. leucotomos in Nonirradiated or Ultraviolet-Radiated Epidermal Keratinocytes
}

\author{
Neena Philips ${ }^{1}$ and Salvador Gonzalez ${ }^{2}$ \\ ${ }^{1}$ School of Natural Sciences, Fairleigh Dickinson University, 1000 River Road, Teaneck, NJ 07666, USA \\ ${ }^{2}$ Memorial Sloan-Kettering Cancer Center, 1275 York Ave, NY 10065, USA \\ Correspondence should be addressed to Neena Philips; nphilips@fdu.edu
}

Received 19 August 2013; Accepted 21 October 2013

Academic Editors: A. B. Salmon, T. B. Shea, A. Shukla, and Y. Yoshida

Copyright (C) 2013 N. Philips and S. Gonzalez. This is an open access article distributed under the Creative Commons Attribution License, which permits unrestricted use, distribution, and reproduction in any medium, provided the original work is properly cited.

\begin{abstract}
There is loss of the structural integrity of the extracellular matrix (ECM) with intrinsic aging as well as photoaging, largely due to reactive oxygen species (ROS). The structural ECM proteins include the microfibrils that are composed of fibrillin. The structural ECM proteins are primarily degraded by the matrixmetalloproteinases (MMPs) and elastase enzymes. The MMPs are inhibited by the tissue inhibitors of MMPs (TIMPs). A primary regulator of the ECM proteins is transforming growth factor- $\beta$ (TGF- $\beta$ ), and the chaperone proteins important for its formation are the heat shock proteins (HSP). P. leucotomos extract beneficially regulates of MMPs, TIMPs, and TGF- $\beta$ in nonirradiated or ultraviolet (UV) radiated fibroblasts and melanoma cells. The hypothesis of this research was that the antioxidant activity or chemistry of $P$. leucotomos extract would also directly inhibit elastase activity, stimulate the cellular expression of TIMPs, fibrillins, and TGF- $\beta$, and regulate HSPs in nonirradiated and UVA or UVB radiated epidermal keratinocytes. P. leucotomos directly inhibited elastase activity, stimulated the cellular expression of TIMPs, fibrillins, and TGF- $\beta$, and differentially regulated HSPs in nonirradiated and UVA or UVB radiated epidermal keratinocytes. We infer that the P. leucotomos extract strengthens the ECM and is effective in the prevention or treatment of intrinsic and photoaging of skin.
\end{abstract}

\section{Introduction}

Cellular oxidative stress from increased reactive oxygen species (ROS) occurs with intrinsic aging, and more so from the exposure of skin to ultraviolet (UV) radiation. The UV radiation includes the long-wavelength UV-A light (320-400 nm), which increases cellular ROS and ROS mediated cellular/extracellular matrix $(\mathrm{ECM})$ damage, and the short-wavelength UV-B light $(290-320 \mathrm{~nm})$, which directly damages DNA as well as cells/ECM through ROS. The ROS facilitate the degradation/remodeling of the ECM that leads to skin aging or cancer. The ECM proteins include the microfibrillar network that is composed of the fibrillin, synthesized by the epidermal keratinocytes and the dermal fibroblasts. The degradation of the ECM proteins is primarily by the matrixmetalloproteinases (MMPs) and elastase, which are inhibited by the cellular inhibitors of MMPs, tissue inhibitors of MMPs (TIMPs). The primary regulator of the ECM is transforming growth factor- $\beta$ (TGF- $\beta$ ). Antioxidants remove ROS, and thereby have the potential to inhibit ROS mediated skin aging or cancer. Polypodium leucotomos is a topical fern plant of the order polypodiaceae. P. leucotomos is rich in polyphenols that are antioxidants and its extract is marketed for topical or systemically photoprotection as Fernblock or Heliocare [1].

P. leucotomos extract inhibits MMP activity directly and the cellular expression of MMPs, while stimulating the expression of TIMPs in dermal fibroblasts and melanoma 
cells $[2,3]$. P. leucotomos extract inhibits the expression of MMPs in epidermal keratinocytes; however, its regulation of elastase activity directly, or cellular expression of TIMPs in epidermal keratinocytes, has not been reported [2]. One of the goals of this research was to extend P. leucotomos photoprotective effects through the direct inhibition of elastase activity and the stimulation of TIMPs in nonirradiated and UVA or UVB radiated epidermal keratinocytes.

The elastin fibers that provide stretch-recoil properties to skin are composed of an elastin core surrounded by fibrillin microfibrils. The fibrillins also form a microfibrillar network at the epidermal-dermal junction. UV radiation causes depletes the fibrillin-rich microfibrils, loss of proper elastin fibers, and their degradation leading to solar elastosis [2]. The stimulation of fibrillin has been used as a marker to screen for photoaging repair agents [4-6]. P. leucotomos extract stimulates the expression of elastin in dermal fibroblasts and keratinocytes [3]. The regulation of fibrillin by P. leucotomos extract has not been reported and was one of the goals of this research.

A primary beneficial regulator of the ECM is transforming growth factor- $\beta$ (TGF- $\beta$ ). It inhibits MMPs and stimulates TIMPs in dermal fibroblasts [3, 7]. However, it can facilitate carcinogenesis [8]. P. leucotomos extract differentially and beneficially regulates TGF- $\beta$ in dermal fibroblasts and melanoma cells, by stimulating TGF- $\beta$ in dermal fibroblasts and inhibiting it in melanoma cells [3]. The expression of TGF- $\beta$ is inhibited with aging and counteracted by UV radiation [9-11]. The regulation of TGF- $\beta$ by P. leucotomos extract in nonirradiated or UV radiated epidermal keratinocytes has not been reported and was studied in this research.

The heat shock proteins (HSP) are induced in response to cellular stress and aid in the stabilization of proteins. The HSP-27 is closely linked to epidermal differentiation and the formation of the epidermis [12]. The HSP-70 has antiinflammatory activity and prevents UVB radiation induced epidermal damage in transgenic mice expressing HSP-70 [13]. Further, the expression of HSP-70 decreases with cellular senescence [14]. The regulation of HSP-27 and HSP-70 by $P$. leucotomos extract in nonirradiated or UV radiated epidermal keratinocytes has not been reported and was studied in this research.

The intrinsic and photoaging of skin, largely mediated by ROS, increases the activity of MMPs and elastase decreases expression of TIMPs, fibrillins, and TGF- $\beta$ and has differential effects on HSPs. The hypothesis of this research was that the antioxidant activity or chemistry of $P$. leucotomos extract would also directly inhibit elastase activity, stimulate the cellular expression of TIMPs, fibrillins, and TGF- $\beta$, and regulate HSPs in nonirradiated and UVA or UVB radiated epidermal keratinocytes

\section{Methods}

2.1. Elastase Calibration and Inhibition. Elastase (Elastin Product Co. no. SE563) was calibrated by reacting 2-fold serial dilutions of the enzyme (starting concentration of
$1 \mu \mathrm{g} / \mu \mathrm{L}$ ) with its substrate (Bachem: I-1270, $0.5 \mathrm{mM}$ ) in incubation buffer $(0.09 \mathrm{M}$ Tris $-0.5 \mathrm{M} \mathrm{NaCl})$. The reaction kinetics was measured fluorometrically (355 excitation/450 emission) every 5 mins for 1.5 hours. The optimal enzyme concentration (linear dose response) was determined to be $0.1 \mu \mathrm{g} / \mathrm{mL}$.

P. leucotomos $(0,0.001 \%$, or $0.01 \%)$ was incubated with the optimal concentration of elastase in incubation buffer for 10 minutes followed by the addition of $0.5 \mathrm{mM}$ elastase substrate. Activity of elastase was measured fluorometrically at 355 excitation/450 emission every 10 minutes for an hour. The initial reading was subtracted from the final reading and the data (fluorescent units about 16,000 for $0 \mathrm{PL}$ ) converted to $\%$ of control (0 PL).

2.2. Cell Culture. Human keratinocytes (Cascade Biologics) in $33 \mathrm{~mm}$ dishes were nonirradiated or UV radiated $(7.5 \mathrm{~mJ}$ UVA or $7.5 \mathrm{~mJ}$ UVB) as previously described [2, 3], Subsequently, cells were dosed with PL $(0,0.01 \%$ or $1 \%)$ for 24 hours. The media were examined fir TIMP-1, TIMP-2, fibrillin-1, fibrillin-2, and TGF- $\beta$ protein levels. The cells were examined for heat shock proteins (HSP-27, 70).

2.3. ELISA. The protein levels were determined by ELISA (Antibodies or kits - Fibrillin: Elastin products Co.; TIMPs: Sigma; TGF- $\beta$ : R\&D Systems; Heat shock proteins: StressGen; Kirkguaard and Perry Laboratories, Inc.). $100 \mu \mathrm{L}$ aliquots of test samples or respective standards were added to a target-specific 96 -well plates for 24 hours at $4^{\circ} \mathrm{C}$. The wells were blocked with bovine serum albumin and then incubated with respective antibodies for 1 hour at room temperature. The plate was washed with wash buffer, incubated with secondary antibody linked to peroxidase for 1 hour at room temperature, washed, and subsequently incubated with peroxidase substrate until color development, measured spectrophotometrically at $405 \mathrm{~nm}$.

2.4. Data Analysis. The data was analyzed for significant difference by ANOVA and student $t$-tests at 95\% confidence interval. The effect UV radiation was analyzed relative to cells that were nonirradiated and not exposed to P. leucotomos extract (control). The effect each of the P. leucotomos concentrations on nonirradiated, UVA-radiated, or UVB-radiated cells was analyzed relative to respective UV radiated cells without $P$. leucotomos extract (0\% PL).

\section{Results}

3.1. P. leucotomos Directly Inhibits Elastase Activity. P. leucotomos extract was directly inhibitory to elastase activity. Relative to control ( 0 additive at $100 \%$ ), the elastase activity was significantly inhibited by $0.001 \%, 0.01 \%$ to $42 \%$, and $26 \%$ by $P$. leucotomos extract $(P<0.05)$ (Figure $1(\mathrm{a}))$.

3.2. P. leucotomos Stimulates TIMP Expression in Keratinocytes. UVA radiation did not significantly alter the expression of TIMP-1 and significantly inhibited TIMP-2 protein levels to $24 \%$ of control $(P<0.05)$ (Figures $1(\mathrm{a})$ 


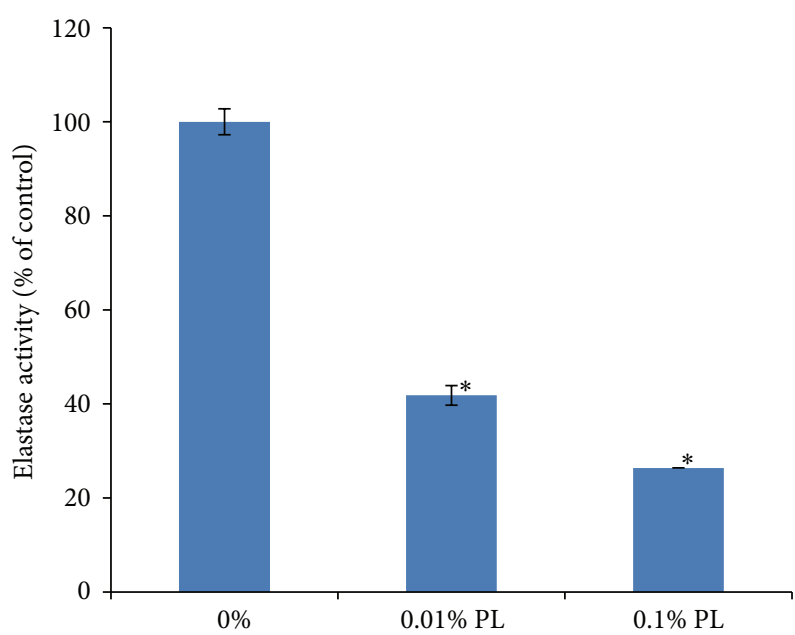

(a)

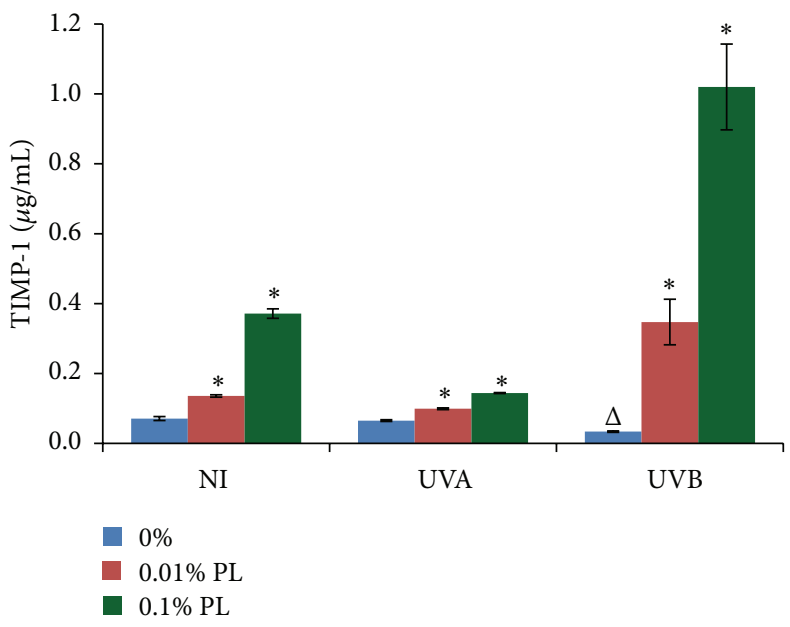

(b)

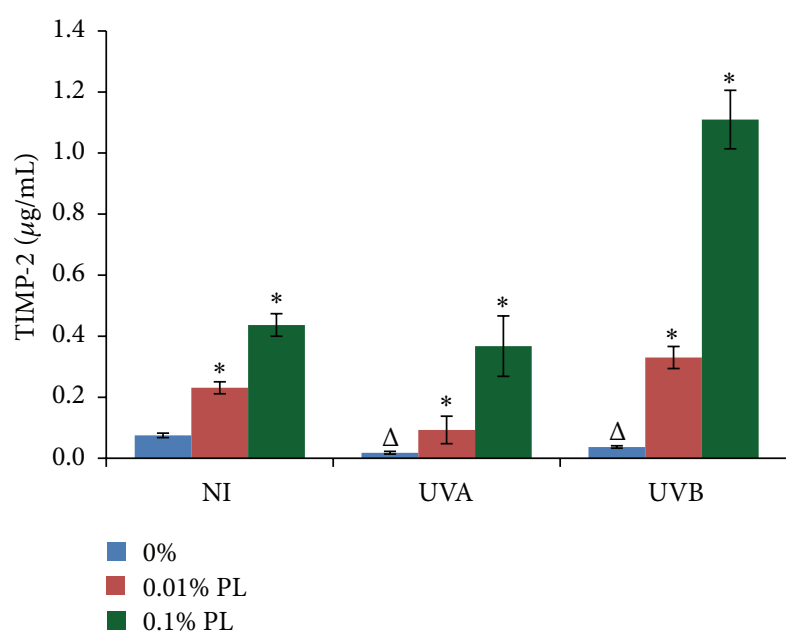

(c)

FIGURE 1: Direct inhibition of elastase activity by P. leucotomos and stimulation of TIMP-1 and TIMP-2 expression by P. leucotomos in epidermal keratinocytes. Elastase was incubated with $0,0.01$, or $0.1 \%$ P. leucotomos (PL) prior to the addition of elastase substrate and the enzyme activity was determined fluorometrically (a). Nonirradiated (NI) UVA or UVB radiated keratinocytes were exposed to PL (0, 0.01\% or $0.1 \%$ ) for 24 hours and examined for TIMP-1 protein (b) and TIMP-2 protein (c). ${ }^{*} P<0.05$, relative to respective controls ( $0 \%$ PL). $\Delta$ : $P<0.05$, between nonirradiated and UV radiated cells. Error bars represent standard deviation, $n=4$.

and 1(b)). UVB radiation significantly inhibited TIMP-1 and TIMP-2 protein levels to $48 \%$ and $49 \%$ of control [TIMP1: $100 \%: 71 \mu \mathrm{g} / \mathrm{mL}$; TIMP-2: $100 \%: 75 \mu \mathrm{g} / \mathrm{mL}](P<0.05)$ (Figures 1(b) and 1(c)).

P. leucotomos significantly stimulated TIMP-1 and -2 expression in nonirradiated and UV radiated keratinocytes. In nonirradiated keratinocytes, the expression of TIMP- 1 and TIMP- 2 was significantly stimulated by P. leucotomos at $0.01 \%$ (TIMP-1: 191\% of control; TIMP-2: 308\% of control) and 0.1\% (TIMP-1: 523\% of control; TIMP-2: 582\% of control) $(P<$ 0.05 ) (Figures $1(\mathrm{~b})$ and $1(\mathrm{c})$ ). In UVA radiated keratinocytes, TIMP-1 and TIMP-2 protein levels were significantly stimulated by P. leucotomos at $0.01 \%$ (TIMP-1: $152 \%$ of UVA respective control; TIMP-2: 517\% of UVA respective control), and $0.1 \%$ (TIMP-1: $220 \%$ of UVA respective control; TIMP2: $2041 \%$ of UVA respective control) $(P<0.05)$ (Figures 1(b) and $1(\mathrm{c})$ ). In UVB radiated keratinocytes, P. leucotomos significantly stimulated TIMP-1 and TIMP-2 protein levels at $0.01 \%$ (TIMP-1: $1028 \%$ of UVB respective control; TIMP-2: $892 \%$ of UVB respective control) and 0.1\% (TIMP-1: 3018\% of UVB respective control; TIMP-2:3000\% of UVB respective control) $(P<0.05)$ (Figures 1(b) and 1(c)).

3.3. P. leucotomos Stimulates Fibrillin Expression in Keratinocytes. UVA radiation significantly inhibited fibrillin1 and -2 protein levels to about $45 \%$ and $48 \%$ of respective controls (Figures 2(a) and 2(b)). UVB radiation did not significantly alter fibrillin-1 expression but significantly inhibited fibrillin-2 protein levels to $69 \%$ of control $(P<0.05)$ (Figures 2(a) and 2(b)).

$P$. leucotomos significantly $(P<0.05)$ stimulated expression of fibrillin-1 and -2 in nonirradiated and UV radiated 


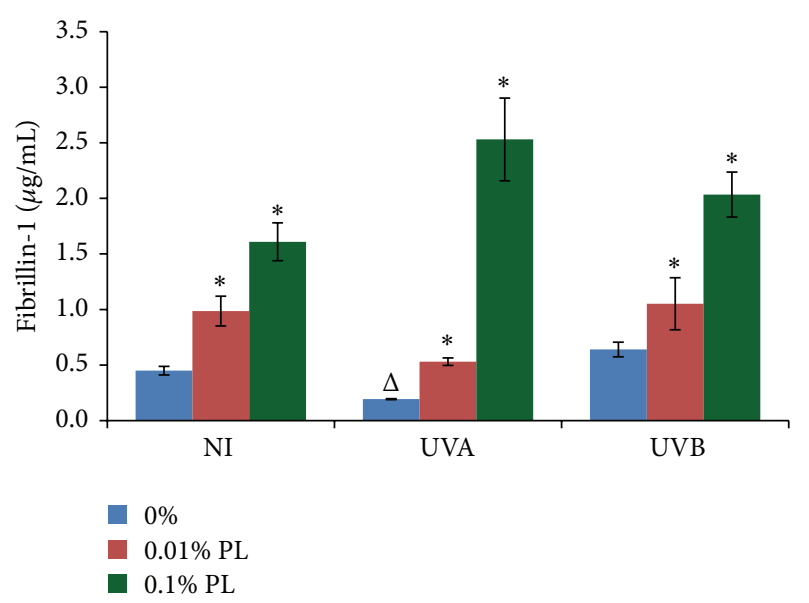

(a)

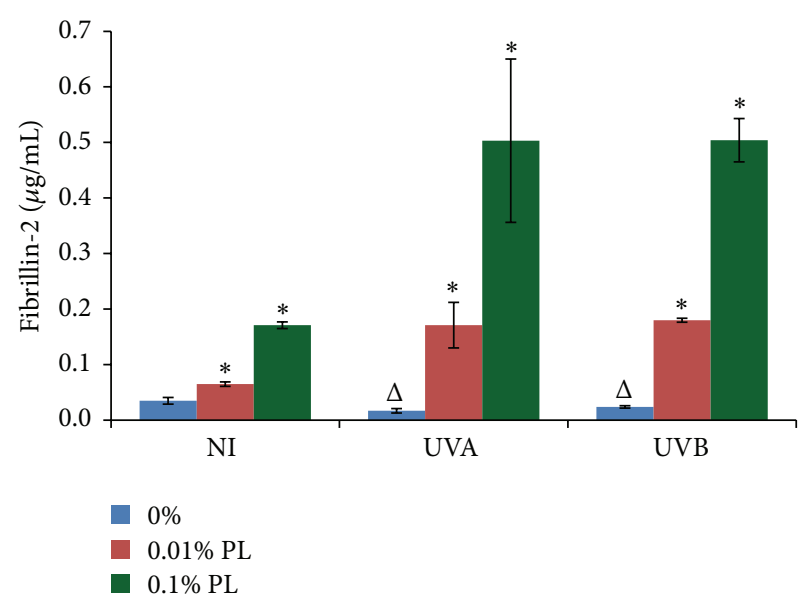

(b)

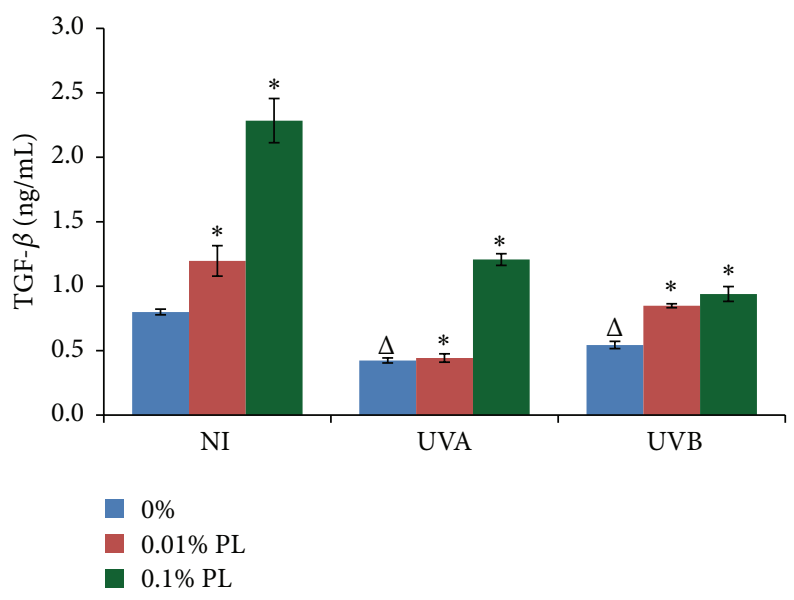

(c)

FIGURE 2: Stimulation of fibrillin-1, fibrillin-2, and TGF- $\beta$ expression by $P$. leucotomos in epidermal keratinocytes. Nonirradiated (NI), UVA, or UVB radiated keratinocytes were exposed to PL $(0,0.01 \%$ or $0.1 \%)$ for 24 hours and examined for fibrillin-1 protein (a), fibrillin-2 protein (b), and TGF- $\beta$ protein (c). ${ }^{*} P<0.05$, relative to respective controls $(0 \%$ PL). $\Delta: P<0.05$, between nonirradiated and UV radiated cells. Error bars represent standard deviation, $n=4$.

keratinocytes (Figures $2(\mathrm{a})$ and $2(\mathrm{~b})$ ). The expression of fibrillin-1 was significantly stimulated in nonirradiated and UV radiated keratinocytes by P. leucotomos at $0.01 \%$ (nonirradiated: $219 \%$ of control; UVA: $274 \%$ of UVA respective control; UVB: $164 \%$ of UVB respective control) and at $0.1 \%$ (nonirradiated: $358 \%$ of control; UVB: $318 \%$ of UVB respective control; UVA: 700\% of UVA respective control) $(P<$ 0.05 ) (Figure 2(a)). Relative to respective controls, $0.01 \%$ and $0.1 \%$ P. leucotomos significantly stimulated fibrillin-2 protein levels to $186 \%$ and $489 \%$ in nonirradiated keratinocytes, to $1006 \%$ and $2959 \%$ in UVA irradiated keratinocytes, and to $750 \%$ and $2100 \%$ in UVB irradiated keratinocytes $(P<0.05)$ (Figure 2(b)).

3.4. P. leucotomos Stimulates TGF- $\beta$ Expression in Keratinocytes. In keratinocytes, UVA and UVB radiation significantly inhibited the expression of TGF- $\beta$ to $68 \%$ and $53 \%$ of control (100\%: $300 \mathrm{pg} / \mathrm{mL})$, respectively $(P<0.05)$ (Figure 2(c)).
P. leucotomos significantly stimulated TGF- $\beta$ expression in nonirradiated and UV radiated keratinocytes $(P<0.05)$ (Figure 2(c)). The expression of TGF- $\beta$ was significantly stimulated by P. leucotomos at $0.01 \%$ in nonirradiated and UVA radiated keratinocytes to about $150 \%$ of respective controls, and at $0.1 \%$ in nonirradiated, UVA, and UVB radiated keratinocytes to $286 \%, 285 \%$, and $172 \%$ of their respective controls $(P<0.05)$ (Figure $2(\mathrm{c}))$.

\subsection{P. leucotomos Stimulates HSP-27 and -70 in Nonirradiated} Keratinocytes and Counteracts Its Induction in UVA Radiated Keratinocytes. In UVA irradiated keratinocytes, HSP 27 was significantly elevated to $283 \%$ and HSP 70 to $217 \%$ of UVA respective controls [HSP-27: 100\%: $8 \mathrm{ng} / \mathrm{mL}$; HSP-70: 100\%: $20 \mathrm{n} / \mathrm{mL}](P<0.05)$ (Figures 3(a) and 3(b)). UVB radiation did not significantly alter the expression of HSP-27 and HSP70 (Figures 3(a) and 3(b)).

P. leucotomos significantly stimulated HSP-27 and HSP70 in nonirradiated keratinocytes; it significantly stimulated 


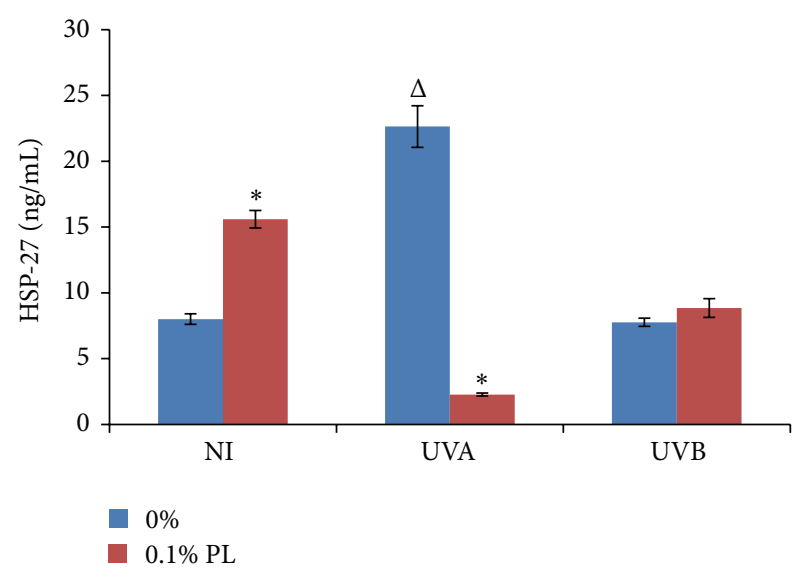

(a)

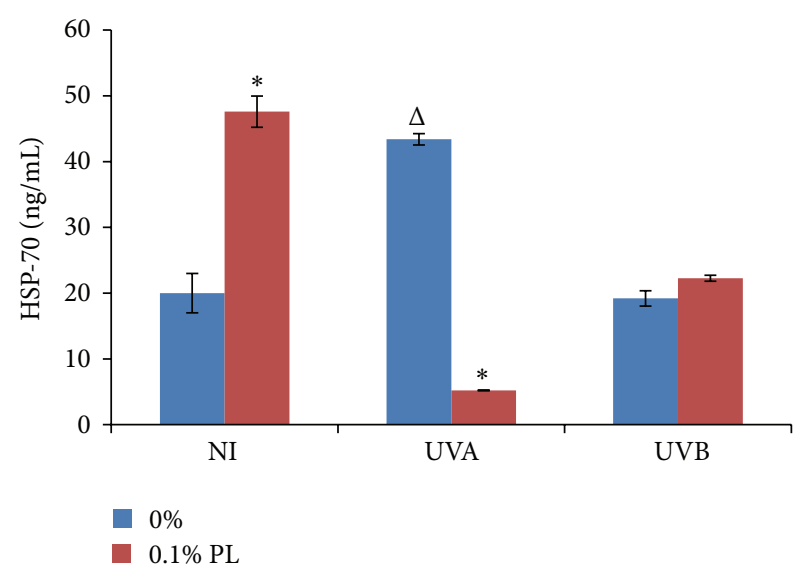

(b)

FIGURE 3: Antagonistic effects of P. leucotomos and UVA radiation on HSP-27 and -70 in epidermal keratinocytes. Nonirradiated (NI), UVA, or UVB radiated keratinocytes were exposed to PL $(0,0.01 \%$ or $0.1 \%)$ for 24 hours and examined for HSP-27 protein (a) and HSP-70 (b). ${ }^{*} P<0.05$, relative to respective controls $(0 \%$ PL). $\Delta: P<0.05$, between nonirradiated and UV radiated cells. Error bars represent standard deviation, $n=4$.

HSP-27 to $195 \%$ of control and HSP-70 to $238 \%$ of control $(P<0.05)$ (Figures 3(a) and 3(b)). P. leucotomos counteracted the stimulation of HSPs in UVA radiated keratinocytes; it inhibited the expression of HSP-27 and HSP-70 to $10 \%$ and $12 \%$ of UVA respective controls, respectively $(P<0.05)$ (Figures 3(a) and 3(b)).

\section{Discussion}

With intrinsic aging or photoaging there is increased activity of MMPs/elastase, reduced expression of TIMPs, fibrillin, and TGF- $\beta$, and altered cellular stress response proteins (HSPs), which collectively compromise the ECM integrity and thereby skin health. The primary cells of the skin that contribute to the ECM integrity are the dermal fibroblasts and the epidermal keratinocytes. The primary mediators of the ECM alterations in aging or photoaging are ROS; and $P$. leucotomos extract with its high polyphenolic antioxidant components beneficially regulates the ECM proteins in nonirradiated or UV radiated dermal fibroblasts [2,3]. We herein present that the P. leucotomos extract directly inhibits elastase activity and beneficially regulates the expression of TIMPs, fibrillin, TGF- $\beta$, and HSPs in nonirradiated UVA or UVB radiated keratinocytes.

The structural integrity of the ECM is compromised with the increase in the basal or induced levels of MMPs/elastase enzymes as well as their activity with aging or exposure to UV radiation. The ECM proteolytic enzymes (MMPs/elastases) are produced by epidermal keratinocytes, dermal fibroblasts, neutrophils and melanoma cells in the mediation of skin aging or cancer $[3,15-18]$. P. leucotomos extract directly inhibits the activities of MMPs (MMP-1, 2, 3, 9) as well as the cellular expression of MMPs (MMP-1, 2) in nonirradiated or UV radiated dermal fibroblasts or epidermal keratinocytes
$[2,3]$. In addition, $P$. leucotomos extract directly inhibits elastase activity and may thereby inhibit the fragmentation of elastin/fibrillin fibers.

Along with the increase in MMPs, there is decrease in TIMPs with aging or photoaging, which increases the cellular MMPs/TIMPs ratio and ECM damage [9-11, 18]. $P$. leucotomos extract stimulates the expression of TIMPs in nonirradiated or UV radiated dermal fibroblasts [3]. It also stimulates the expression of TIMP-1 and TIMP-2 in nonirradiated and UVA or UVB radiated keratinocytes, suggesting its potential to also prevent the loss of structural or microfibrillar ECM proteins with aging or exposure to UV radiation through its effects on keratinocytes.

The fibrillins compose the microfibrils that provide the scaffold for the assembly of the elastin fibers and a network at the epidermal-dermal junction as well as the dermis. There are three known fibrillin proteins; fibrillin-1 is most abundant in mature tissue and fibrillin-2 is associated with elastin fibers $[19,20]$. The microfibrils are degraded by proteases or reduced in expression at the epidermal junction and in the elastin fibers with aging or photoaging [4-6]. The induction of fibrillin is associated with skin repair, and is a target in the screening for cosmetic products $[5,6]$. P. leucotomos extract stimulates the expression of elastin in nonirradiated or UVA or UVB radiated fibroblasts and keratinocytes [2]. It also stimulates the expression of fibrillin- 1 and -2 in nonirradiated and UVA or UVB radiated keratinocytes, suggesting the strengthening of microfibrils and the elastin fibers.

A major regulator of the ECM proteins is TGF- $\beta$, which stimulates the expression of TIMPs and fibrillin $[10,18,21]$. UV radiation counteracts the expression or effect of TGF- $\beta$ in dermal fibroblasts, and keratinocytes $[10,11]$. P. leucotomos stimulates TGF- $\beta$ expression in nonirradiated and UV radiated fibroblasts [3]. It also stimulates the expression of TGF- $\beta$ expression in nonirradiated UVA or UVB radiated 
keratinocytes; suggesting that the stimulation of TIMPs and fibrillins by $P$. leucotomos may be mediated through its induction of TGF- $\beta$ expression.

The chaperone proteins that are important in the formation of the ECM proteins as well for cell health are the HSPs. The HSP-27 is considered the marker for epidermal differentiation and HSP-70 for anti-inflammatory activity [12-14]. There is loss of expression of HSPs with aging, whereas they are induced in carcinogenesis [12-14, 22]. $P$. leucotomos extract counteracts the induction of HSP-27, 70 and 90 as well as MMP-1 by the growth inhibiting but metastasis supporting ascorbate dose in melanoma cells [22]. P. leucotomos extract induces the expression of HSP-27 and HSP-70 in nonirradiated keratinocytes, suggesting facilitation in the formation of the ECM proteins as well as improved cell health. The UVA radiation stimulated HSP-27 and HSP70 , which was counteracted by counteracted the P. leucotomos extract that may suggest the inhibition of the cellular stress response in these cells. The UVB radiated keratinocytes, with or without $P$. leucotomos extract, did not show alterations in the HSP-27 or HSP-70 in comparison with nonirradiated keratinocytes, suggesting a different mechanism in the UVB radiated cells than in UVA cells that may also have prevented the intrinsic stimulation of HSPs by the P. leucotomos extract. While the primary mechanism of UVA radiation is through the stimulation of ROS, the primary mechanism of UVB radiation is direct DNA damage and secondarily through ROS.

\section{Conclusion}

P. leucotomos directly inhibits elastase activity, stimulates the cellular expression of TIMPs, fibrillins, and TGF- $\beta$, and differentially regulates HSPs in nonirradiated and UVA or UVB radiated epidermal keratinocytes. In combination with its effects on nonirradiated or UVA or UVB radiated fibroblasts, we infer that $P$. leucotomos extract (Fernblock) is effective in the strengthening of the ECM integrity and thereby the prevention or treatment of intrinsic and photoaging of skin.

\section{Acknowledgment}

Neena Philips is grateful to the assistance of Marvin Tuason and David Bynum from Fairleigh Dickinson University and Hyendo Hwang, Rosemaire Arena, and Donna Leonardi from Bergen Academies.

\section{References}

[1] L. Gombau, F. García, A. Lahoz et al., "Polypodium leucotomos extract: antioxidant activity and disposition," Toxicology in Vitro, vol. 20, no. 4, pp. 464-471, 2006.

[2] N. Philips, J. Smith, T. Keller, and S. Gonzalez, "Predominant effects of Polypodium leucotomos on membrane integrity, lipid peroxidation, and expression of elastin and matrixmetalloproteinase-1 in ultraviolet radiation exposed fibroblasts, and keratinocytes," Journal of Dermatological Science, vol. 32, no. 1, pp. 1-9, 2003.
[3] N. Philips, J. Conte, Y.-J. Chen et al., "Beneficial regulation of matrixmetalloproteinases and their inhibitors, fibrillar collagens and transforming growth factor- $\beta$ by Polypodium leucotomos, directly or in dermal fibroblasts, ultraviolet radiated fibroblasts, and melanoma cells," Archives of Dermatological Research, vol. 301, no. 7, pp. 487-495, 2009.

[4] R. E. B. Watson, C. E. M. Griffiths, N. M. Craven, C. A. Shuttleworth, and C. M. Kielty, "Fibrillin-rich microfibrils are reduced in photoaged skin. Distribution at the dermalepidermal junction," Journal of Investigative Dermatology, vol. 112 , no. 5, pp. 782-787, 1999.

[5] R. E. B. Watson, N. M. Craven, S. Kang, C. J. P. Jones, C. M. Kielty, and C. E. M. Griffiths, "A short-term screening protocol, using fibrillin-1 as a reporter molecule, for photoaging repair agents," Journal of Investigative Dermatology, vol. 116, no. 5, pp. 672-678, 2001.

[6] R. E. Watson, S. Ogden, L. F. Cotterell et al., "Effects of a cosmetic "anti-ageing" product improves photoaged skin [corrected]," The British Journal of Dermatology, vol. 161, no. 2, pp. 419-426, 2009.

[7] N. Philips, T. Keller, and S. Gonzalez, “TGF $\beta$-like regulation of matrix metalloproteinases by anti-transforming growth factor$\beta$, and anti-transforming growth factor- $\beta 1$ antibodies in dermal fibroblasts: implications for wound healing," Wound Repair and Regeneration, vol. 12, no. 1, pp. 53-59, 2004.

[8] N. Philips and N. Onwubalili, "Anti transforming growth factor-beta (TGF- $\beta$ ) increases the expressions of matrix metalloproteinase-1 (MMP-1) and growth factors in a renal adenocarcinoma cell line," BIOS, vol. 73, pp. 86-90, 2002.

[9] G. Zeng, H. M. McCue, L. Mastrangelo, and A. J. T. Millis, "Endogenous TGF- $\beta$ activity is modified during cellular aging: Effects on metalloproteinase and TIMP-1 expression," Experimental Cell Research, vol. 228, no. 2, pp. 271-276, 1996.

[10] N. Philips, R. Arena, and S. Yarlagadda, "Inhibition of ultraviolet radiation mediated extracellular matrix remodeling in fibroblasts by transforming growth factor-b," BIOS, vol. 80, article 1, 2009.

[11] N. Philips, "An anti TGF- $\beta$ antibody increased the expression of transforming growth factor- $\beta$, matrix metalloproteinase-1, and elastin, and its effects were antagonized by ultraviolet radiation in epidermal keratinocytes," Journal of Dermatological Science, vol. 33, no. 3, pp. 177-179, 2003.

[12] C. Jonak, M. Mildner, G. Klosner et al., "The hsp27kD heat shock protein and $\mathrm{p} 38$-MAPK signaling are required for regular epidermal differentiation," Journal of Dermatological Science, vol. 61, no. 1, pp. 32-37, 2011.

[13] M. Matsuda, T. Hoshino, Y. Yamashita et al., "Prevention of UVB radiation-induced epidermal damage by expression of heat shock protein 70," Journal of Biological Chemistry, vol. 285, no. 8 , pp. $5848-5858,2010$.

[14] A. Gutsmann-Conrad, A. R. Heydari, S. You, and A. Richardson, "The expression of heat shock protein 70 decreases with cellular senescence in vitro and in cells derived from young and old human subjects," Experimental Cell Research, vol. 241, no. 2, pp. 404-413, 1998.

[15] F. Rijken, R. C. M. Kiekens, and P. L. B. Bruijnzeel, "Skininfiltrating neutrophils following exposure to solar-simulated radiation could play an important role in photoageing of human skin," British Journal of Dermatology, vol. 152, no. 2, pp. 321-328, 2005. 
[16] N. Tsuji, S. Moriwaki, Y. Suzuki, Y. Takema, and G. Imokawa, "The role of elastases secreted by fibroblasts in wrinkle formation: implication through selective inhibition of elastase activity," Photochemistry and Photobiology, vol. 71, pp. 283-290, 2001.

[17] J. Labat-Robert, A. Fourtanier, B. Boyer-Lafargue, and L. Robert, "Age dependent increase of elastase type protease activity in mouse skin effect of UV-irradiation," Journal of Photochemistry and Photobiology B, vol. 57, no. 2-3, pp. 113-118, 2000.

[18] N. Philips, T. Keller, C. Hendrix et al., "Regulation of the extracellular matrix remodeling by lutein in dermal fibroblasts, melanoma cells, and ultraviolet radiation exposed fibroblasts," Archives of Dermatological Research, vol. 299, no. 8, pp. 373-379, 2007.

[19] H. Zhang, W. Hu, and F. Ramirez, "Developmental expression of fibrillin genes suggests heterogeneity of extracellular microfibrils," Journal of Cell Biology, vol. 129, no. 4, pp. 1165-1176, 1995.

[20] F. Ramirez and L. Pereira, "The fibrillins," International Journal of Biochemistry and Cell Biology, vol. 31, no. 2, pp. 255-259, 1999.

[21] H. H. Kim, S. Cho, S. Lee et al., "Photoprotective and anti-skinaging effects of eicosapentaenoic acid in human skin in vivo," Journal of Lipid Research, vol. 47, no. 5, pp. 921-930, 2006.

[22] N. Philips, L. Dulaj, and T. Upadhya, "Growth inhibitory mechanism of ascorbate and counteraction of its matrix metalloproteinases-1 and transforming growth factor-beta stimulation by gene silencing or P. leucotomos," AntiCancer Research, vol. 29, pp. 3233-3238, 2008. 


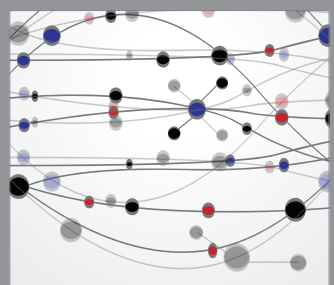

The Scientific World Journal
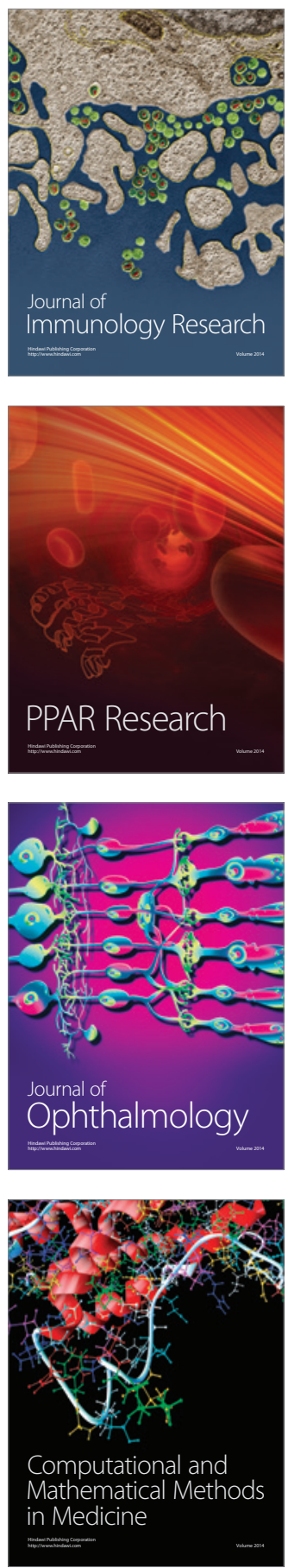

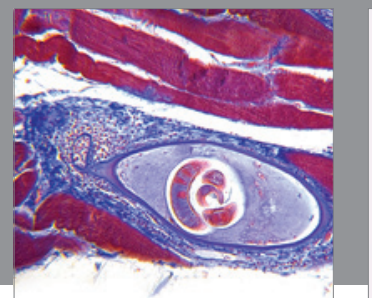

Gastroenterology

Research and Practice
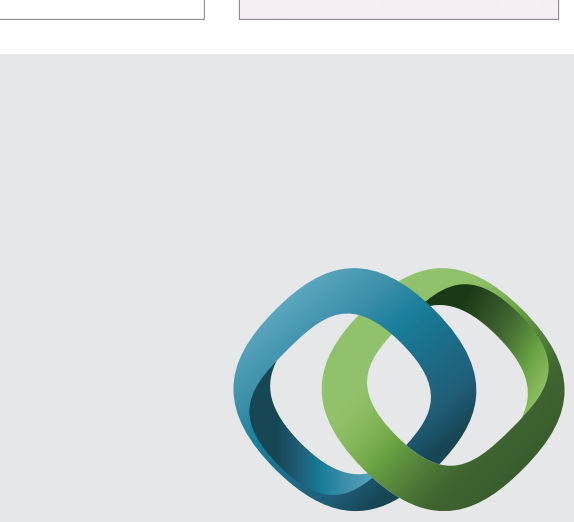

\section{Hindawi}

Submit your manuscripts at

http://www.hindawi.com
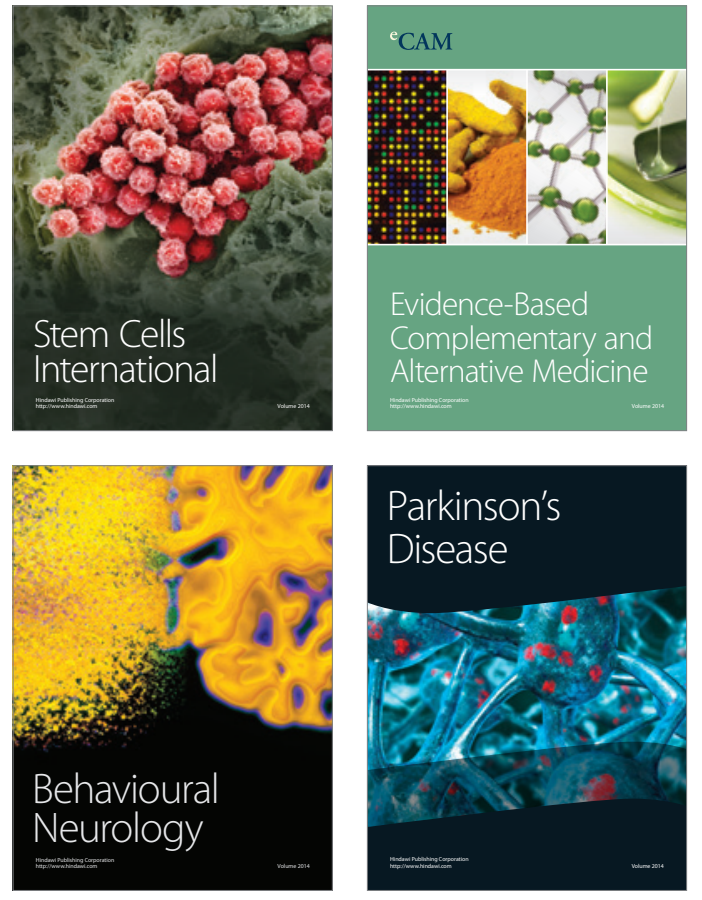
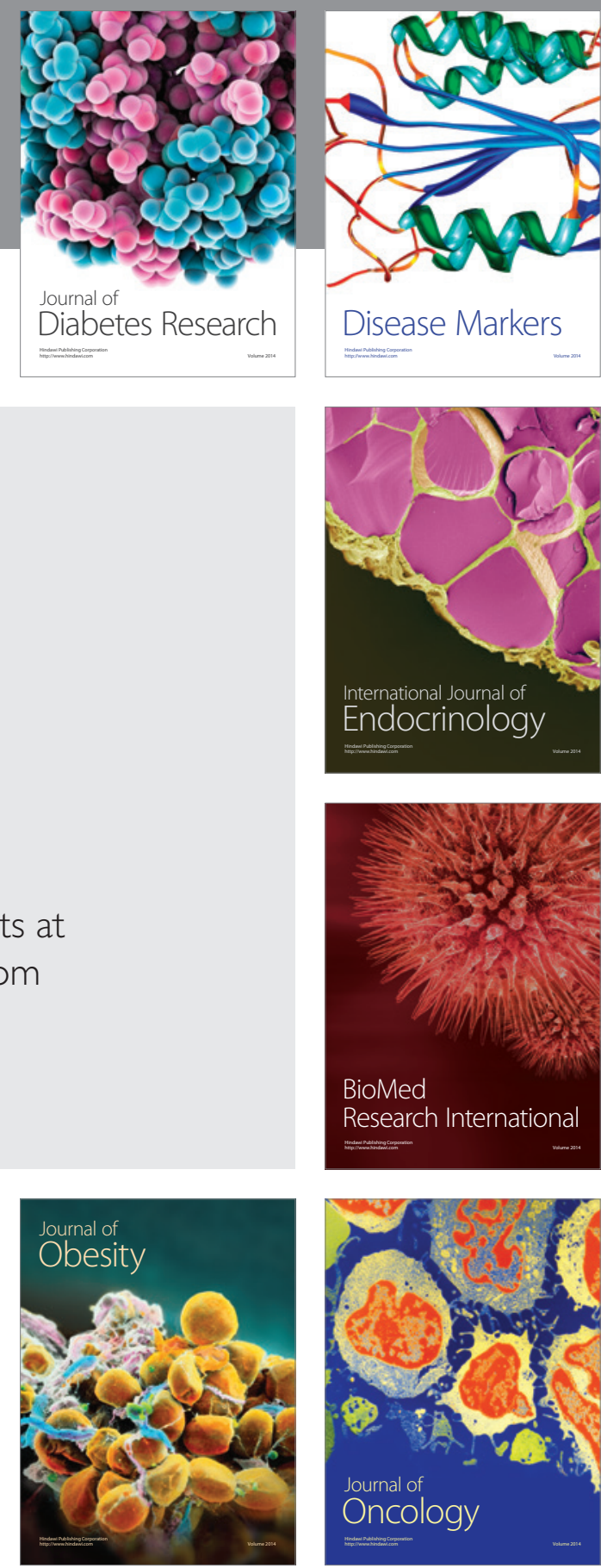

Disease Markers
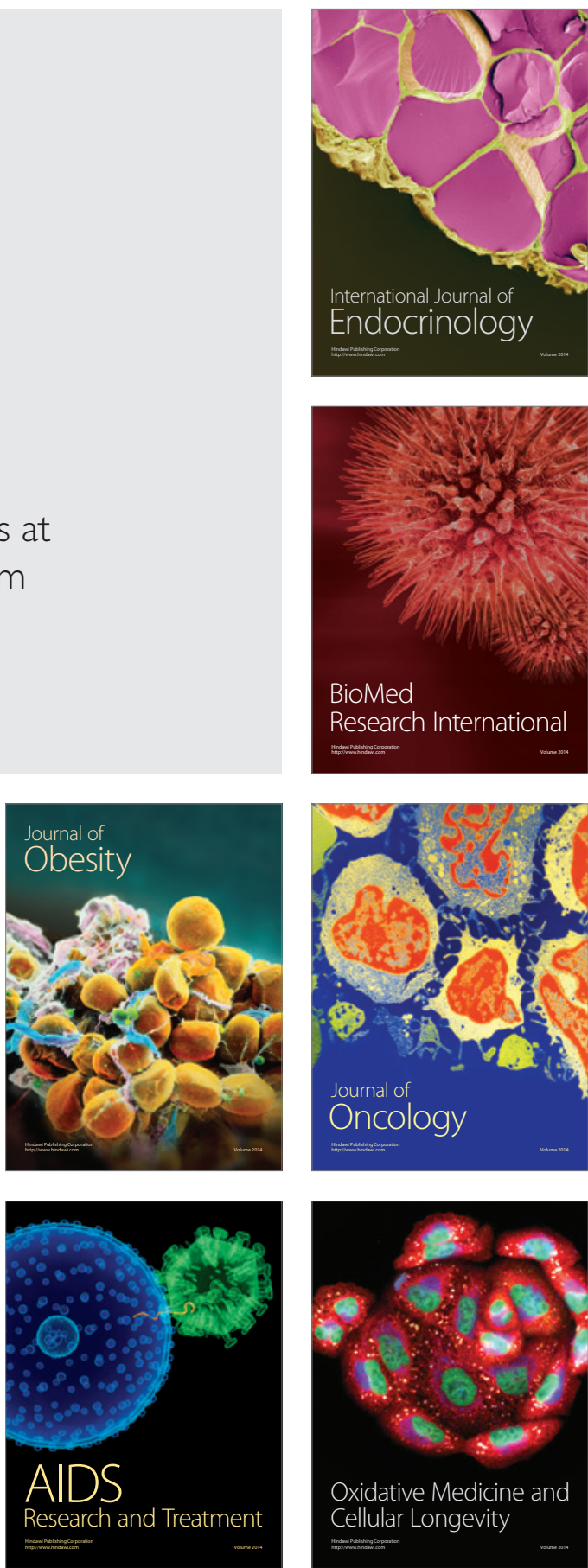